
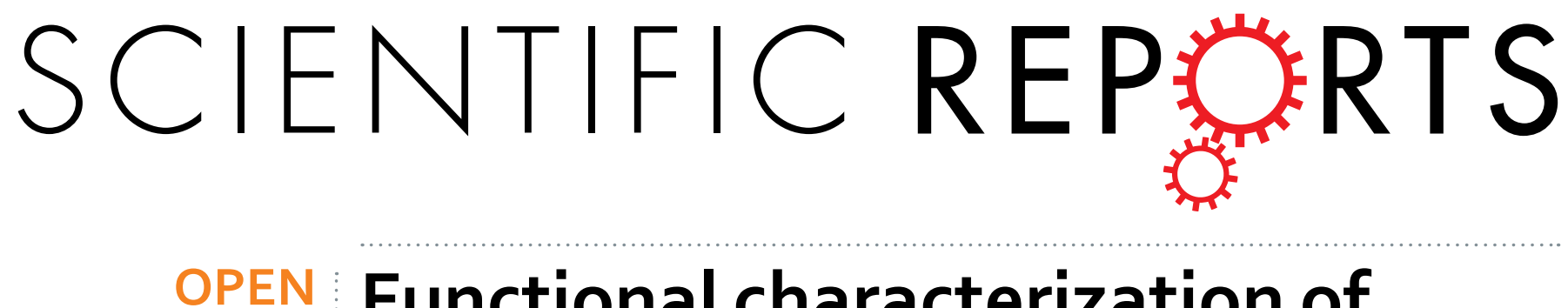

\title{
Functional characterization of
} \section{the late embryogenesis abundant} (LEA) protein gene family from

Received: 23 September 2015

Accepted: 14 December 2015

Published: 19 January 2016

\section{Pinus tabuliformis (Pinaceae) in Escherichia coli}

\author{
Jie Gao ${ }^{1} \&$ Ting Lan ${ }^{2}$
}

Late embryogenesis abundant (LEA) proteins are a large and highly diverse gene family present in a wide range of plant species. LEAs are proposed to play a role in various stress tolerance responses. Our study represents the first-ever survey of LEA proteins and their encoding genes in a widely distributed pine (Pinus tabuliformis) in China. Twenty-three $L E A$ genes were identified from the $P$. tabuliformis belonging to seven groups. Proteins with repeated motifs are an important feature specific to $L E A$ groups. Ten of 23 pine $L E A$ genes were selectively expressed in specific tissues, and showed expression divergence within each group. In addition, we selected 13 genes representing each group and introduced theses genes into Escherichia coli to assess the protective function of PtaLEA under heat and salt stresses. Compared with control cells, the $E$. coli cells expressing PtaLEA fusion protein exhibited enhanced salt and heat resistance and viability, indicating the protein may play a protective role in cells under stress conditions. Furthermore, among these enhanced tolerance genes, a certain extent of function divergence appeared within a gene group as well as between gene groups, suggesting potential functional diversity of this gene family in conifers.

Abiotic stresses, such as drought, salinity, and extreme temperature are limiting factors for normal plant growth and development. High salinity upsets homeostasis in water potential and ion distribution at both cellular and whole plant levels ${ }^{1}$. It results in molecular damages, arrest of development and even mortality ${ }^{2}$. High temperature can cause irreversible damages including protein denaturation, aggregation and degradation ${ }^{3}$, as well as protein synthesis inhibition and enzymes inactivation. Extreme temperature can also increase fluidity of membrane lipids ${ }^{4}$ which could lead to loss of membrane integrity ${ }^{5-7}$. Higher plants have developed several genetic responses to these environmental factors ${ }^{8}$. An important group is the late embryogenesis abundant $(L E A)$ protein gene family which functions in cellular protection during abiotic stress tolerance ${ }^{9,10}$. Since the first discovery of the LEA proteins in cotton and wheat ${ }^{11,12}$, the LEA proteins have been described throughout the entire plant kingdom, as well as in other organisms, from invertebrate to prokaryotes ${ }^{13,14,15}$.

The $L E A$ genes are mainly expressed in seeds and, as its name suggests, are accumulated during late embryo development stage, comprising up to $4 \%$ of cellular proteins ${ }^{16}$. They have also been found in seedlings, roots and other organs throughout the whole developmental stage. After suffering environmental stresses, plants accumulate high levels of LEA proteins in response ${ }^{17,18}$. They have been proposed to have various functions, including protection of cellular structures from the effects of water loss and desiccation ${ }^{19}$, protection of proteins from stress-induced damage $\mathrm{e}^{19,20,21}$, sequestration of ions ${ }^{20}$, and folding of denatured proteins ${ }^{22}$. LEA proteins can also act as chaperone proteins to resist cellular damage ${ }^{18,23}$.

The LEA genes often exist as a large gene family in higher plants. For example, Arabidopsis and Populus contain 51 and 53 members respectively, which are divided into eight groups based on the amino acid sequence homology

${ }^{1}$ Key Laboratory of Tropical Forest Ecology, Xishuangbanna Tropical Botanical Garden, Chinese Academy of Sciences, Menglun, Yunnan, China. ${ }^{2}$ State Key Laboratory of Systematic and Evolutionary Botany, Institute of Botany, Chinese Academy of Sciences, Beijing 10093, China. Correspondence and requests for materials should be addressed to J.G. (email: gaojie@xtbg.org.cn) 
and specific motifs ${ }^{24}$. To date, comprehensive analysis of LEA genes have only been made in angiosperms like Arabidopsis thaliana ${ }^{25}$, Oryza sativa ${ }^{26}$, Hordeum vulgare ${ }^{27}$, Prunus mume ${ }^{28}$, Populus $^{24}$, Solanum ${ }^{29,30}$, Malus domestica ${ }^{31}$ and legumes ${ }^{32}$. In contrast, considerably fewer $L E A$ genes have been identified in gymnosperms, mostly due to limited genome information. Only a few LEAs and LEA-like proteins have been examined in Picea glauca ${ }^{33}$, Pseudotsuga menziesii ${ }^{34}$ and Pinus pinaster ${ }^{35}$.

Pinus tabuliformis, a dominant conifer species is widely distributed over mountainous areas from northern to central China. It grows from 0-2700 $\mathrm{m}$ above sea level. Most of the region has heterogeneous habitats, including a wide range of temperatures, altitudes, elevated ozone and UV levels ${ }^{36}$. Long generation time and large effective population sizes of $P$. tabuliformis suggest that this species has adapted to variable environmental conditions. Gymnosperms, especially conifers represent a large group of plants with a long evolutionary history. However, long generation time makes it a challenge to investigate transgenic functioning in conifers. The accumulation of LEAs during environmental stresses also makes the study of a single LEA gene difficult. Therefore, an alternative approach is to express the PtaLEA proteins in vivo in yeast or E.coli for functional expression screening, which has been successful in previous studies ${ }^{37-41}$. By integrating phylogenetic analysis, protein motif structure, gene expression and heterologous expression analysis in E. coli of 23 full-length LEA genes, we examine the evolutionary and functional diversity of the LEA gene family in P. tabuliformis.

\section{Materials and Methods}

Identification of LEA genes from the Pinus taede EST database. We identified LEA genes from Pinus taeda by searching 53 Arabidopsis LEA protein sequences in the P. taeda EST database (328,662 ESTs in the National Center for Biotechnology Information database: http://www.ncbi.nlm.nih.gov/) using TBLASTN. All the identified $P$. taeda candidates were analyzed using the protein family database (Pfam; http://pfam.sanger. ac.uk/) to confirm the presence of $L E A$ conserved domains in their protein structure. We retrieved 43 whole $L E A$ genes from $P$. taeda, allowing us to design 43 primers based on these gene sequences and use them to clone the LEA genes from $P$. tabuliformis.

Molecular cloning, phylogenetic analysis and bioinformatic analysis of Pinus tabuliformis LEA genes. Total RNA isolation from seeds of P. tabuliformis was performed using an Aurum Total RNA Kit (Bio-Rad, Hercules, CA, USA). Total RNA was treated with RNase-free DNase I (Promega, Madison, WI, USA) and the first strand cDNA was synthesized using an RNA PCR Kit (AMV) version 3.0 (TaKaRa, Otsu, Japan). PCR conditions consisted of an initial denaturation of $3 \mathrm{~min}$ at $94^{\circ} \mathrm{C}$, followed by $35 \mathrm{cycles}$ of $30 \mathrm{~s}$ at $94^{\circ} \mathrm{C}, 40 \mathrm{~s}$ at $55^{\circ} \mathrm{C}$ and $60 \mathrm{~s}$ at $72^{\circ} \mathrm{C}$, and a final extension of $5 \mathrm{~min}$ at $72^{\circ} \mathrm{C}$. PCR products were cloned into $p E A S Y$-T3 (TransGen, Beijing, China), and sequenced in both directions to verify the gene sequence. We confirmed that the sequences obtained had LEA conserved domains using Pfam.

We cloned 23 full-length $L E A$ genes from P. tabuliformis. The multiple sequence alignment of the 23 PtaLEA genes was performed using MUSCLE ${ }^{42}$ (http://www.ebi.ac.uk/Tools/msa/muscle/) with default parameters and manually adjusted by BioEdit ${ }^{43}$. Phylogenetic analysis was carried out by the maximum parsimony method with 1000 bootstrap replicates using MEGA v. 5 software ${ }^{44}$.

Conserved motifs for each LEA proteins were investigated using the Multiple Expectation maximization for Motif Elucidation (MEME) system version $4.10 .24^{45}$ (http://meme.sdsc.edu/meme/cgi-bin/meme.cgi), any number of repetitions are expect to be distributed in sequences, with the minimum width of 6 , and maximum width of 50. The signal peptide analysis was done by the TargetP algorithm ${ }^{46}$ (TargetP: http://www.cbs.dtu.dk/services/ TargetP/).

Transcription abundance of PtaLEA genes in $\boldsymbol{P}$. tabuliformis tissues. To determine the expression pattern of each P. tabuliformis LEA gene, specific primers of the 23 LEA genes were designed (Table S-2) and used in RT-PCR, based on the multiple sequence alignment of $L E A$ gene sequences. Total RNA was isolated from the top bud, needle, phloem in the stem, root of more than 10-year old P. tabuliformis and also two weeks germinants. Total RNA was treated as in the previously described method. Amplification was performed in a volume of $25 \mu \mathrm{L}$ containing $10 \mathrm{pmol}$ of each primer, $2.5 \mu \mathrm{L}$ TaKaRa $10 \times$ PCR buffer, $0.125 \mu \mathrm{L} \mathrm{TaKaRa} \mathrm{ExTaq}(5 \mathrm{U} / \mu \mathrm{L}), 2 \mu \mathrm{L} \mathrm{dNTP}$ ( $2.5 \mathrm{mM}$ each). PCR conditions consisted of an initial denaturation of $3 \mathrm{~min}$ at $94^{\circ} \mathrm{C}$, followed by $25 \mathrm{cycles}$ of $30 \mathrm{~s}$ at $94^{\circ} \mathrm{C}, 40 \mathrm{~s}$ at $60^{\circ} \mathrm{C}$ and $1 \mathrm{~min}$ at $72^{\circ} \mathrm{C}$ with a final extension of $3 \mathrm{~min}$ at $72^{\circ} \mathrm{C}$. In all PCR analysis, the Actin gene was used as an internal control. The PCR products were analyzed by $1.5 \%$ agarose gel electrophoresis. PCR products from each sample were validated by DNA sequencing. For each biological sample three repetitions were performed.

Construction of E.coli strains expressing LEA proteins. First, we added a NotI site upstream from the start codon ATG with the 5' end primer, and a SpeI site after the stop codon TAA with the $3^{\prime}$ end primer. Then, the LEA genes we cloned from the first step were used to do the PCR amplification. The PCR products were ligated into T-vector (Promega Madison, USA). The vector was then digested using a NotI/SpeI double digestion, and the resulting DNA was gel-purified and subcloned into the TWIN1 vector (New England BioLabs, Beijing, China), linearized by a double digestion with the same restriction enzymes to construct TWIN-LEA plasmid. The PtaLEA coding region and the junction sequences were confirmed by DNA sequencing. The TWIN-LEA plasmid was transformed into applicable E. coli host strain BL21 (DE3). Transformed E. coli cells were grown in LuriaBertani (LB) medium containing $100 \mathrm{mg} / \mathrm{ml}$ of carbenicillin and kept at $37^{\circ} \mathrm{C}$ overnight. The overnight cultures were diluted 1,000-fold using fresh $\mathrm{LB}$ medium and incubation continued at $37^{\circ} \mathrm{C}$ until mid-log phase $(3-4 \mathrm{~h}$, $\mathrm{OD}_{600}=0.6$ ). Isopropyl b-D-thiogalactopyranoside (IPTG) was then added to a final concentration of $1 \mathrm{mM}$, and incubation was continued at $37^{\circ} \mathrm{C}$ for $3 \mathrm{~h}$. Cells were harvested by centrifugation and resuspended in Buffer 1 


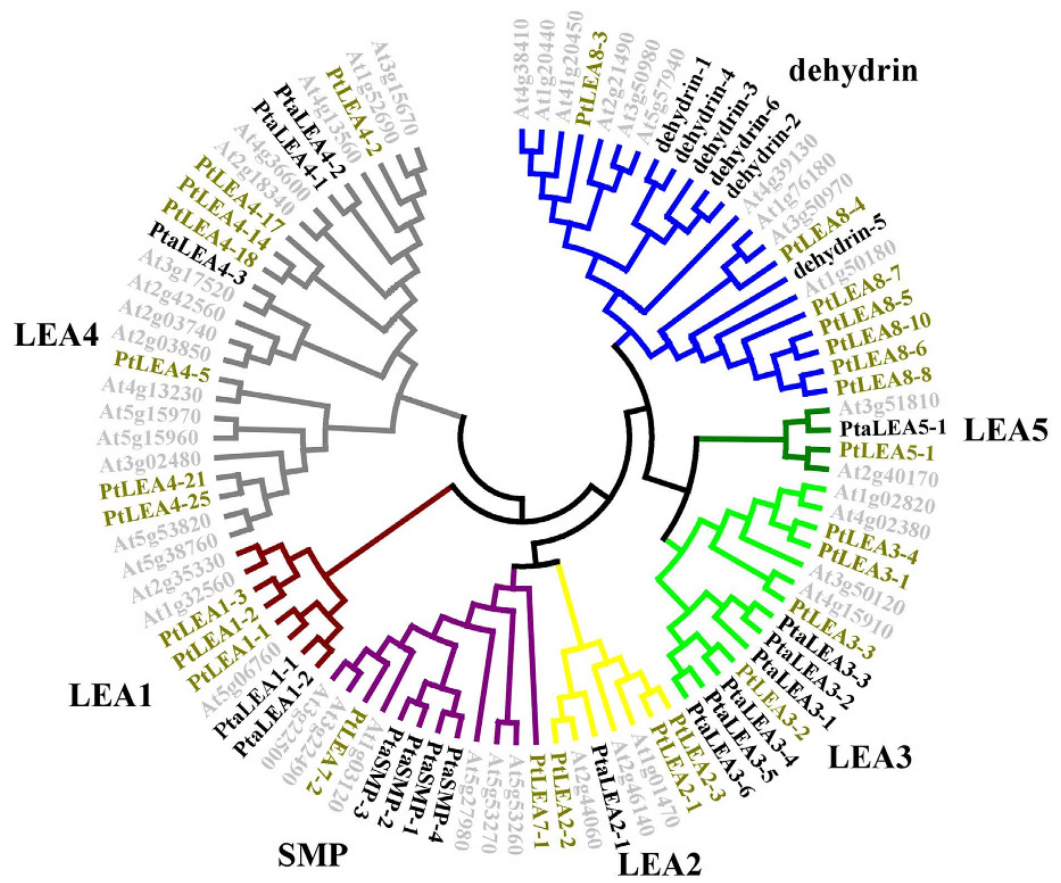

Figure 1. Phylogenetic relationships of Pinus tabuliformis, Populus and Arabidopsis LEA genes. LEA groups are distinguished by color. The light grey represented the LEA genes form Arabidopsis, the bold names represented the LEA genes from $P$. tabuliformis, and the green names are genes from Populus.

[20 mM Tris- $\mathrm{HCl}$ (pH 8.5), $500 \mathrm{mM} \mathrm{NaCl}, 1 \mathrm{mM}$ EDTA] and lysed by sonication. The lysate was centrifuged at $12,000 \mathrm{rpm}$ for $10 \mathrm{~min}$ to collect clarified cell extract for SDS-PAGE analysis.

Heat and salt treatment with transformed E.coli cells. Cell cultures were grown as described above. IPTG was added to mid-log phase cultures $\left(\mathrm{OD}_{600}=0.6\right)$ to a final concentration of $1 \mathrm{mM}$, and incubation was continued at $37^{\circ} \mathrm{C}$ for $3 \mathrm{~h}$. After IPTG induction, the cultures were diluted and transferred to $50^{\circ} \mathrm{C}$. Samples $(10 \mu \mathrm{l})$ were taken at $0,30,60,120$, and $180 \mathrm{~min}$, and serial dilutions were plated onto LB plus carbenicillin plates. We have detected the values of $\mathrm{OD}_{600}$ from each sample and make them to be the same value. Cell viability was estimated by counting the number of colony-forming units after incubation of the plate over- night at $37^{\circ} \mathrm{C}$. For salt treatment, after IPTG induction, ten microliters of each sample was spotted onto the LB plates(and we also controlled the $\mathrm{OD}_{600}$ for each of these samples),containing $171 \mathrm{mM} \mathrm{NaCl}$ and the $\mathrm{LB}$ plates with $300 \mathrm{mM} \mathrm{NaCl}$, $400 \mathrm{mM} \mathrm{NaCl}, 500 \mathrm{mM} \mathrm{NaCl}, 600 \mathrm{mM} \mathrm{NaCl}, 700 \mathrm{mM} \mathrm{NaCl}$, respectively. Plates were then incubated at $37^{\circ} \mathrm{C}$ over night.

The colony number on each plate was recorded using the software of Cellprofiler ${ }^{47}$. Viability ratio of the transformants under salt and heat conditions was calculated according to the following formula:

$$
\begin{aligned}
\text { Viability ratio }= & (\text { mean of colony number on stressed Plate } \\
& / \text { mean of colony number oncontrol LB plate }) \times 100 \% .
\end{aligned}
$$

For both treatments (heat and salt), the means of three experiments were determined from three independent transformants.

\section{Results}

Sequence and structure characteristics of the PtaLEA gene. Forty-three full-length genes encoding putative LEA proteins in $P$. taeda were identified from the Pinus taeda EST database. Due to the lack of Pinus whole-genome sequence information, we designed the primers based on the sequences of $P$. taeda. The $P$. tabuliformis may have the same or even more members of LEA proteins but we successfully cloned 23 full length $L E A$ genes from the P. tabuliformis. All of these genes had $L E A$ conserved domains which was confirmed by the Pfam domain analysis. A phylogenetic analysis based on amino acid sequences of the predicted proteins revealed that the 23 PtaLEA genes grouped into seven groups together with the Arabidopisis and Populus LEAs, confirming that they belonged to the $L E A$ family (Fig. 1). We followed the $L E A$ gene family nomenclature in Hundertmank and Hincha (2008) and the Pfam domain name. We found that group LEA3 and dehydrin, each have six genes. The $L E A 1$ group has two members, $L E A 4$ has three members, and SMP has four members, whereas groups LEA2 and $L E A 5$ each contained only one gene. We did not obtain any members from LEA6 group.

These genes and their families possessed several group-specific characteristics (Table 1; Fig. 2). A majority of $P$. tabuliformis LEA proteins contained repeated motifs that were often specific to a LEA group. We analyze the group-specific characteristics with the comparison to the conserved motifs indentified in Arabidopsis ${ }^{48}$ and other species ${ }^{49,50}$. The LEA4 group had several repeats of conserved motif 1 : three times in PtaLEA4-1, up 


\begin{tabular}{|l|c|c|c|c|c|}
\hline Groups & $\begin{array}{c}\text { Pfam } \\
\text { groups }\end{array}$ & Proteins & $\begin{array}{c}\text { Protein } \\
\text { length(aa) }\end{array}$ & $\begin{array}{c}\text { Molecular } \\
\text { weights(kD) }\end{array}$ & $\begin{array}{c}\text { Predicted subcellular } \\
\text { localization }\end{array}$ \\
\hline LEA1 & LEA1 & PtaLEA1-1 & 114 & 12.55 & other \\
\hline & & PtaLEA1-2 & 117 & 12.51 & other \\
\hline LEA2 & LEA2 & PtaLEA2-1 & 190 & 21.40 & other \\
\hline LEA3 & LEA3 & PtaLEA3-1 & 113 & 12.22 & Mitochondrion \\
\hline & & PtaLEA3-2 & 96 & 10.72 & Mitochondrion \\
\hline & & PtaLEA3-3 & 106 & 11.80 & Mitochondrion \\
\hline & & PtaLEA3-4 & 104 & 11.07 & Mitochondrion \\
\hline & & PtaLEA3-5 & 103 & 11.31 & Mitochondrion \\
\hline & & PtaLEA3-6 & 89 & 9.81 & Mitochondrion \\
\hline LEA4 & LEA4 & PtaLEA4-1 & 153 & 16.02 & other \\
\hline & & PtaLEA4-2 & 98 & 10.44 & other \\
\hline & & PtaLEA4-3 & 314 & 33.99 & other \\
\hline LEA5 & LEA5 & PtaLEA5-1 & 246 & 26.96 & Chloroplast \\
\hline SMP & SMP & PtaSMP-1 & 270 & 27.63 & other \\
\hline & & PtaSMP-2 & 262 & 27.46 & other \\
\hline & & PtaSMP-3 & 282 & 29.46 & other \\
\hline & & PtaSMP-4 & 261 & 26.60 & other \\
\hline dehydrin & Dehydrin & dehydrin-1 & 177 & 18.88 & other \\
\hline & & dehydrin-2 & 165 & 18.10 & other \\
\hline & & dehydrin-3 & 238 & 25.85 & other \\
\hline & & dehydrin-4 & 135 & 14.62 & other \\
\hline & & dehydrin-5 & 93 & 10.32 & other \\
\hline & & dehydrin-6 & 161 & 17.68 & other \\
\hline
\end{tabular}

Table 1. The characteristics of the P.tabuliformis LEA proteins.

A

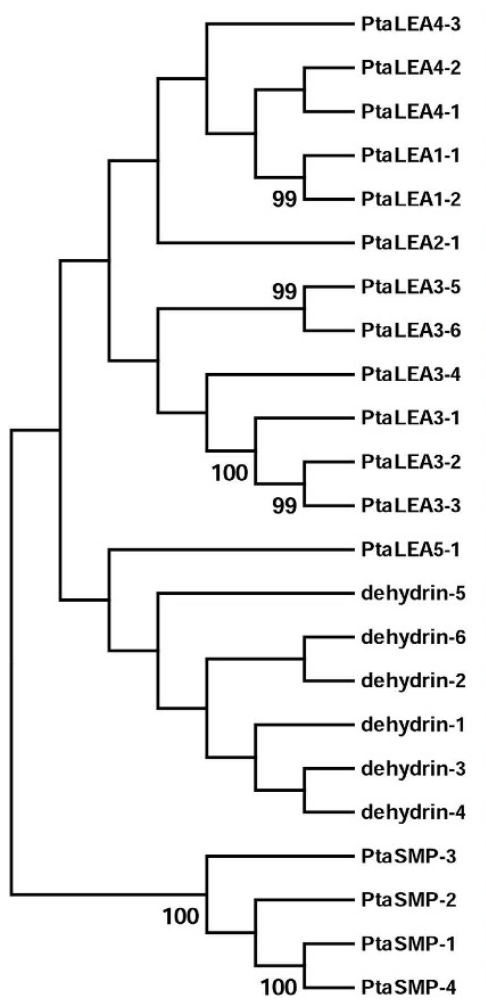

B C

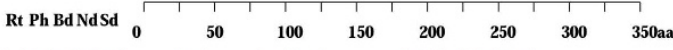

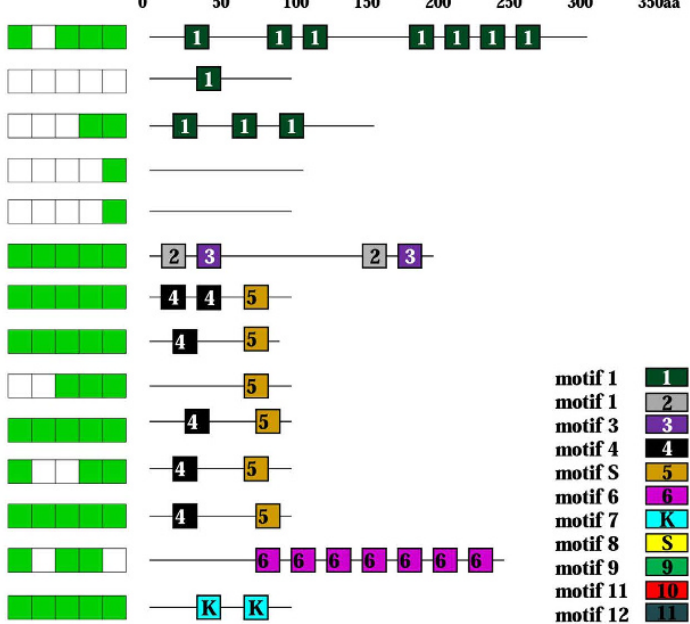

$-9-5-\mathrm{k}-\mathrm{k}-$

[9- $\mathbb{S}-$

$-9-\sqrt{\mathrm{S}}-\mathrm{K}-$

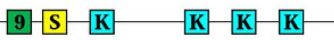

$-\mathbf{s}-\mathbf{K}-\mathbf{K}-\mathbf{k}$

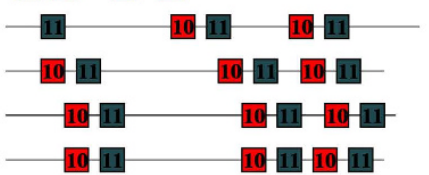

Figure 2. Phylogenetic relationships (A), expression patterns (B) and motif structure of P. tabuliformis LEAs. In $B$, the green box indicates positive detection of gene expression in the corresponding tissue under root (Rt), phloem $(\mathrm{Ph})$, bud $(\mathrm{Bd})$, needle $(\mathrm{Nd})$, two weeks germinants $(\mathrm{Ge})$. In $\mathrm{C}$, boxes labeled with numbers are protein motifs; the motif sequences are provided in the supplemental Fig. 1. 


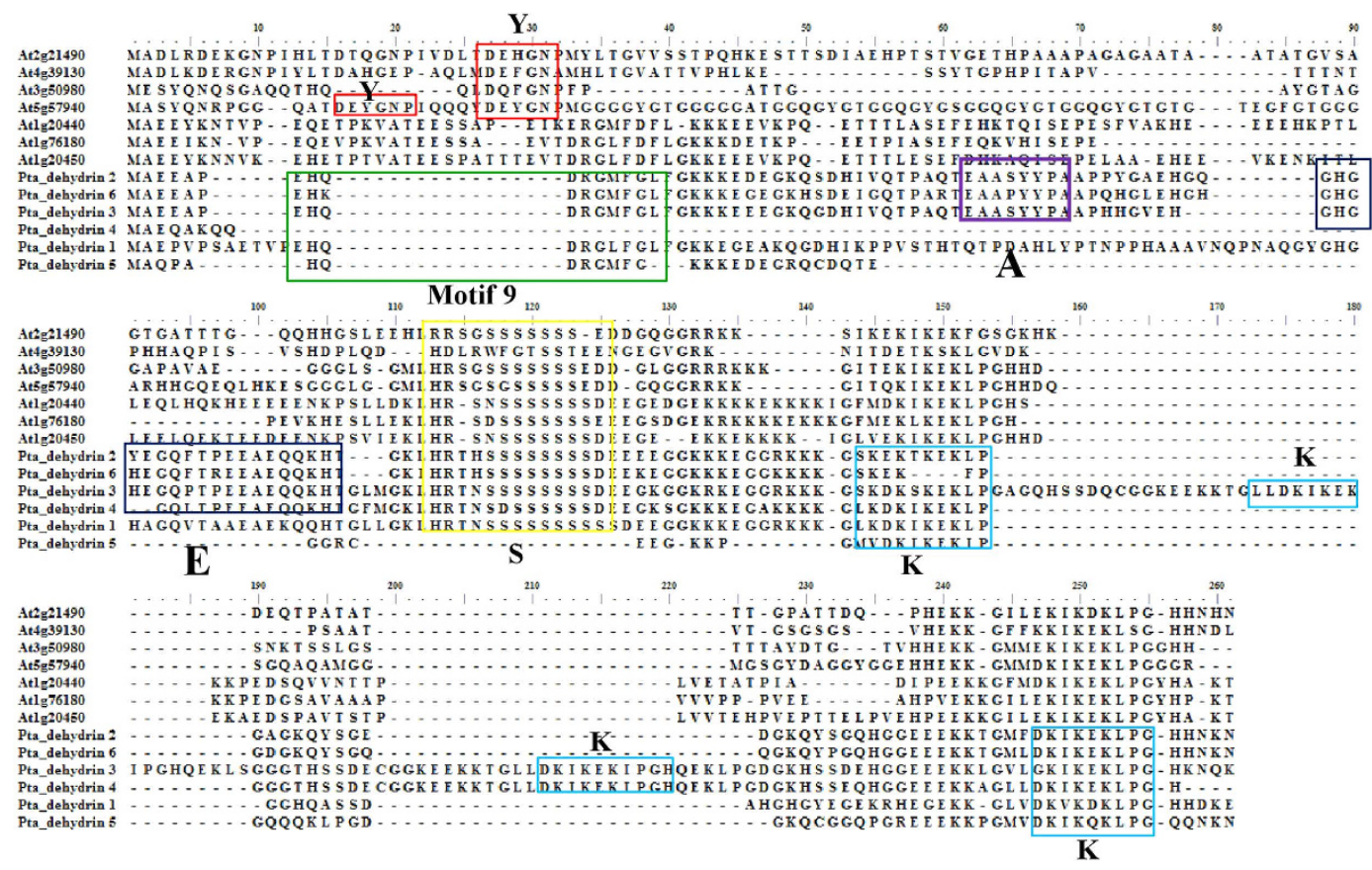

Figure 3. Alignment of the amino-acid sequences of $P$. tabuliformis dehydrins and previously known dehydrin genes from Arabidopsis. Alignments were performed using the CLUSTAL v program. Dashes indicate where a sequence has been expanded to allow optimal sequence alignment. The Y-, S-, K-, A- and E-segments are indicated by color boxes.

to seven times in PtaLEA4-3 but only once in PtaLEA4-2. The PtaLEA5-1 protein from group LEA5 had the repeated group-specific motif 6 with seven repetitions. Group dehydrin is the second largest group in $L E A$ family of which most gene members have been cloned in P. tabuliformis (6 members: dehydrin-1/2/3/4/5/6). MEME analysis showed that the motif structure was variable in this group. All of the six genes share the similar motifs $\mathrm{S}$ and $\mathrm{K}$ which are the conserved motif characteristic to dehydrin proteins, but we failed to find the $\mathrm{Y}$ motif in $P$. tabuliformis which could be found in Arabidopsis (see the alignment in Fig. 3). Apart from that, the repetitions of the motif $\mathrm{K}$ are variable in different dehydrin proteins: twice in dehydrin-5/6/1, three times in dehydrin-4, and up to four times in dehydrin-3, but only appeared once in dehydrin-2. The variation in motif structure indicates functional divergence.

Computational prediction of the subcellular distribution of the LEA proteins using targetP indicates further differences between the LEA groups. Whereas the members of the most groups are localized in the cytosol ("other" in Table 1 indicates that no signal peptide was detected), the LEA3 proteins are exclusively targeted to mitochondria, and the LEA5 is probably targeted to chloroplasts.

Transcription abundance of LEA genes in $P$. tabuliformis. In order to get a better understanding of the expression pattern of $P$. tabuliformis LEA genes, five different tissues/organs (root, phloem, needle, bud and two weeks germinants; Fig. 1B) were collected and analyzed using RT-PCR to detect the transcription abundance. Among the 23 PtaLEA genes, 11 (47.8\%) genes were expressed in all tissues/organs examined under normal condition, indicating that $L E A$ gene family has a wide distribution and expression in $P$. tabuliformis, and are undoubtedly involved in normal plant growth and development. All the genes (except PtaLEA4-2, PtaLEA5-1 and PtaSMP-3) are expressed in the analyzed germinants, indicating that $L E A$ genes have played an indispensable role in the seedling development process of $P$. tabuliformis. Two genes (PtaLEA4-2 and PtaSMP-3) were not expressed in any tissue we studied, possibly because they are expressed at sub-detectable levels, or they are only induced in response to treatments and/or in tissues not including in our study (like seed). The other $10 \mathrm{LEA}$ genes were expressed selectively in specific tissues and showed expression divergence within each group. For example, in the dehydrin group, the genes PtaLEA 8-2/3/4/5 were expressed in all tissues, while dehydrin-6 was expressed in all tissues except the phloem, and dehydrin-1 was only expressed in the root and germinants. Within LEA3 group, the PtaLEA3-1/2/5/6 were expressed in all tissues, while PtaLEA3-3 was expressed in root, needle and germinants, and the PtaLEA3-4 was expressed in bud, needle and germinants. In group LEA4, the PtaLEA4-1 was expressed in needle and germinants, while the PtaLEA4-3 was expressed in all tissues except phloem, however the PtaLEA4-2 did not have detectable expression in any organs. Generally, the different expression pattern of LEA family probably implies the divergence of function between genes within groups.

Enhancement of heat and salt-tolerance of recombinant E.coli cells with PtaLEA genes. In order to determine the function of expressed LEA fusion protein in stress conditions, we selected 2-3 genes from 


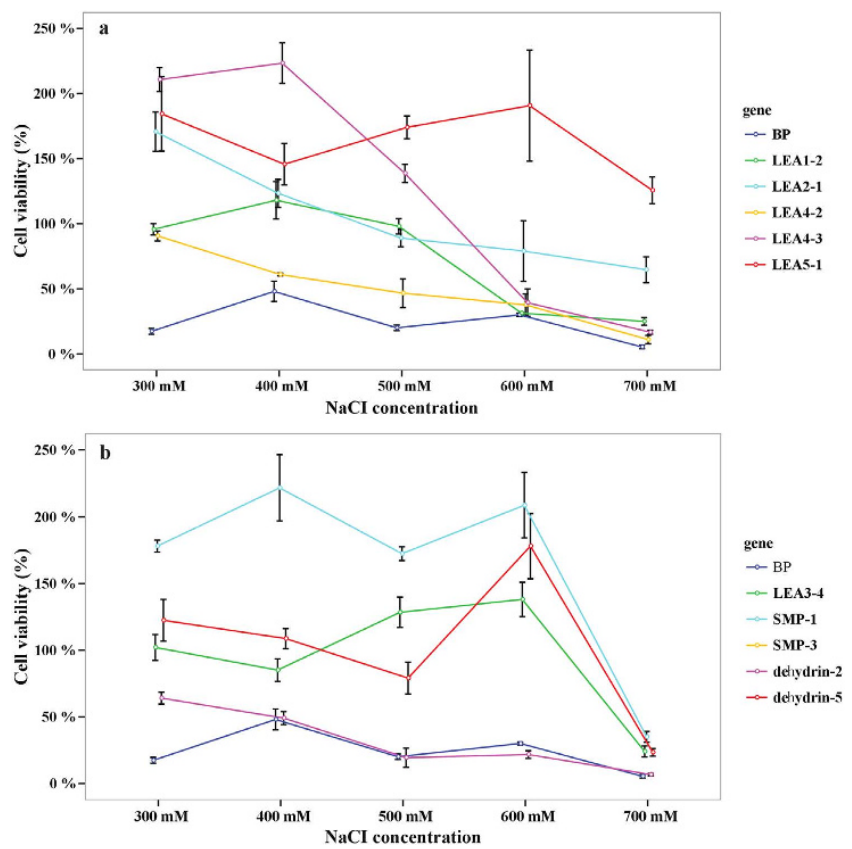

Figure 4. Cell Viability ratio of E.coli transformed with TWIN-LEA and TWIN1 constructs under salt treatment. The values are the mean \pm SE from three samples.

each group except group LEA 2 and LEA5 which we only have one gene in these group (13 genes in total) to construct the recombinant BL/LEA to do the heat and salt treatment.

For the salt treatment, we considered the gene as improving salt tolerance when the viability ratio was higher than the control strain BL/PTWN. The results showed that ten of the thirteen PtaLEA genes had mean viability ratios 2-7 fold higher than those of the control strain BL/PTWN under different concentration of salt stress (Fig. 4a,b). These ten genes belong to different groups. We selected PtaLEA3-3/4, PtaLEA4-2/3 and PtaSMP-1/3 from each group. All these genes, except PtaLEA3-3, have shown an increased salt tolerance. For the group dehydrin, we detected three genes dehydrin-4/5/2, two of which (dehydrin-5/2) have shown an increased salt tolerance with a mean viability ratio 4.3 and 1.3 fold higher than the control strain respectively. LEA2 and LEA5 have only one gene in each group, and LEA1 has two members. We chose all members in these three groups for experiments. The results showed that all of them increased salt tolerance except PtaLEA1-1. The genes PtaSMP-1 (6.8 fold), PtaLEA2-1 (3 fold), PtaLEA4-3 (5.3 fold), PtaLEA5-1 (6.8 fold), dehydrin-5 (4.3 fold) had significantly higher survival ratios than other genes under all concentrations. Genes within group showed different patterns to salt stress, i.e. the viability ratios of PtaLEA4-3 (5.3 fold), dehydrin-5 (4.3 fold) and PtaSMP-1 (6.8 fold) were higher than their group members PtaLEA4-2 (2.0 fold), dehydrin-2 (1.3 fold) and PtaSMP-3 (1.6 fold) respectively.

Results in the heat treatment demonstrated that seven of the thirteen genes showed signs of increased heat tolerance, with viability ratios higher than the control strain BL/PTWN (Fig. 5a,b). Each group of LEA1, LEA5 and $S M P$ had one gene and group $L E A 3$ and dehydrin had two members. After $50^{\circ} \mathrm{C}$ induction for 60 minutes, the viability ratio increased significantly which indicated that the genes need a certain induction time. The PtaLEA3-4 (2.0 fold), dehydrin-4/2 (1.5/2.4 fold) and PtaSMP-3 (2.4 fold) showed the highest viability ratio among these genes. Within LEA3 group, the viability ratio of PtaLEA3-4 (2.0 fold) is much higher than PtaLEA3-2 (1.3 fold).

In conclusion, the expressed $P$. tabuliformis LEA fusion polypeptide conferred salt and heat tolerance to the host cells. Some of the groups showed an increased resistance to heat and salt, but not significantly, like dehydrin-2 that increased the viability by 1.3 fold under salt stress and PtaLEA3-2 that increased the viability by 1.3 fold under heat stress. Some of the groups showed responses to both heat and salt stresses, but genes within group showed different patterns. In particular, within group dehydrin, the dehydrin-5 ( 4.3 fold) showed higher viability under salt treatment, dehydrin-4 (1.5 fold) showed higher viability under heat treatment, while dehydrin-2 responded to both heat ( 2.4 fold) and salt ( 1.3 fold) treatment. All these results suggested that function divergence appeared not only between groups but also between genes within groups.

\section{Discussion}

The LEA genes play crucial roles during embryonic development and response to abiotic stresses throughout the plant kingdom as well as in many other organisms. Our molecular evolution analysis detected 43 gene members in the Pinus taeda genome, fewer than in Populus ${ }^{24}$ which has 53 members. In this study, we have cloned 23 genes from the P. tabuliformis genome belonging to seven groups of the $L E A$ gene family, the protein sequence identities among groups is very lower than the values within each group which exhibited a high degree of divergence among the protein groups. 


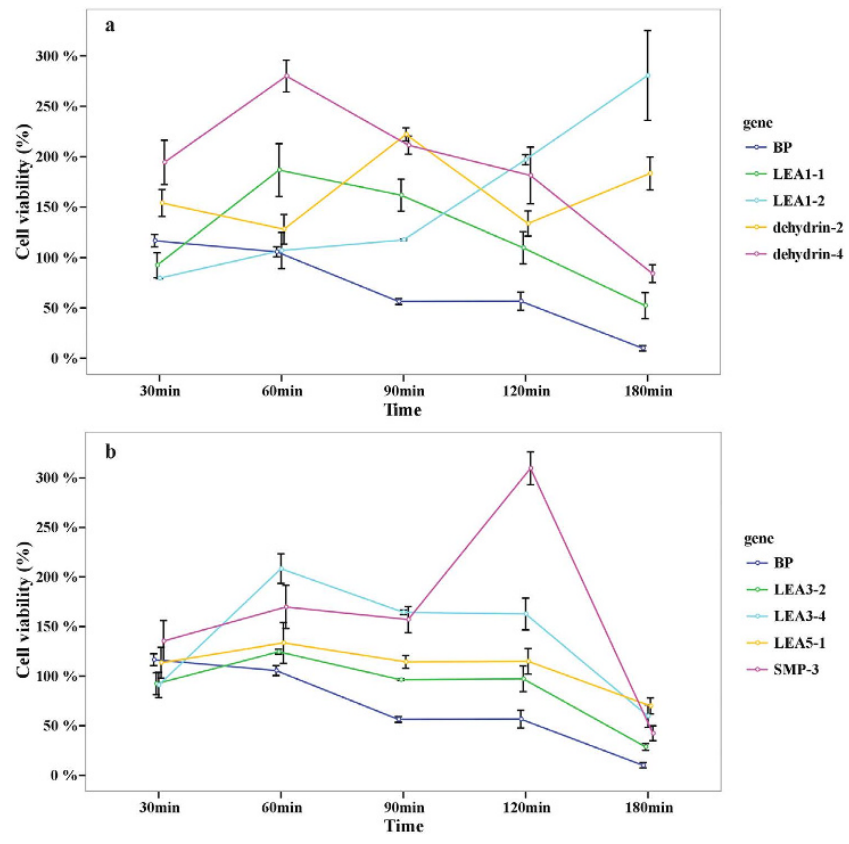

Figure 5. Cell viability ratio of E.coli transformed with TWIN-LEA and TWIN1 constructs under heat treatment at the temperature of $50^{\circ} \mathrm{C}$. The values are the mean $\pm \mathrm{SE}$ from three samples.

Previously, the functional expression screening of LEA proteins in vivo in yeast or E.coli under abiotic stresses have been investigated successfully in various flowering plant species. Wang et al. (2008) investigated an LEA gene from Tamarix expressed in yeast which showed enhanced tolerance to high temperature, $\mathrm{NaHCO}_{3}$, ultraviolet radiation, salt, drought and freezing. Reportedly, one of $L E A 3$ proteins (HVA1) displayed increased tolerance to salt stress and water deficit in both yeast expression system and transgenic rice plants ${ }^{51,52}$. Similarly, one LEA4 gene from Brassica napus is able to enhance the cellular tolerance to temperature and salt stresses of E. coli cells ${ }^{53}$. More recently a SMP protein from tea has reported function as a chaperone enhanced tolerance to E. coli against stresses ${ }^{40}$. However, only a few LEA proteins increasing the tolerance to abiotic stresses had been studied and were primarily from the major group LEA3, LEA4 and dehydrin. No gymnosperm LEA genes had been screened prior to this study. Furthermore, there was no study about the molecular mechanism of $P$. tabuliformis adapting to stressful environments. The P. tabuliformis has a very large distribution area including high temperate, arid and semiarid regions ${ }^{36}$. The gradual salinization of land and the increased summer forest fires have been identified as the major threats to the pine forest in these regions ${ }^{54-56}$. A study showed that Pinus pinea seed germination is sensitive to drought, salinity and heat influence ${ }^{57}$. And another report also confirmed that the reduction of dehydrin proteins in seed have reduced seed longevity in Arabidopsis thalian $a^{58}$. This might be the same in the $P$. tabuliformis seed germination. The LEAs are considered playing a pivotal role in the late embryonic development during the seed germination ${ }^{16}$. In this study, $70 \%$ and $50 \%$ of the PtaLEAs analyzed increased the tolerance to salt and heat stresses, respectively. These genes come from almost all groups of the $L E A$ gene family, which indicates the whole gene family has a function in stress response. Thus, for the first time we have confirmed that the $P$. tabuliformis LEA proteins from all LEA subgroups enhance tolerance to elevated salt and heat conditions. It indicates that the $L E A$ genes have a wider repertoire of roles in stress responses and may play a common role in plant acclimation to stress conditions. This could be due to their highly conserved sequence motifs and high content of hydrophilic amino acids ${ }^{21}$, and we will discuss it later. Furthermore, the LEA subgroups represented diversified adaptation to heat and salt stress. LEA1 increased the resistance to heat and salt, but not significantly, its viability ratio showed 2.3 and 3.1 fold higher than the control strain for heat and salt treatments respectively; while $S M P$, LEA4 and LEA5 induced a higher tolerance to salt stress (more than fivefold higher than the control strain), and $L E A 3$, dehydrin induced a higher tolerance to heat treatment (more than twofold than the control strain). For large gene families with multiple subgroups, genes from different groups usually show functional diversification ${ }^{59}$ Another functional analysis performed using the $E$. coli heterologous expression system, including three genes from group $L E A 1, L E A 3$ and dehydrin respectively, also supported the hypothesis that genes from $L E A 1$ and LEA3 increased the tolerance to salt and low temperature, while genes from dehydrin did not induce obvious growth improvement ${ }^{60}$. While a recent study has investigated functions of six groups of LEA proteins from Arabidopsis thaliana which were expressed in Saccharomyces cerevisiae under desiccation stress. None of proteins from LEA1, LEA5 and AtM showed protection but proteins from LEA2, LEA4 and dehydrin has enhanced tolerance to desiccation stress ${ }^{41}$.

In addition to the expected functional divergence between gene groups, we observed substantial divergence between genes within a single group. The phenomenon was especially apparent in group LEA4 and group dehydrin. LEA4 is the largest group in LEA gene family, with the Populus and Arabidopsis genomes containing 26 and 16 members, respectively ${ }^{24,25}$. It is reported that this group is very heterogeneous and the proteins differ greatly in 
size and GRAVY ${ }^{25}$. LEA4 is also the largest group (16 genes) in P. taeda. In P. tabuliformis we cloned three members and investigated two of them under stress conditions. Within group LEA4, the gene PtaLEA4-3 induced a higher salt tolerance (5.3 fold) than the gene PtaLEA4-2 (2.0 fold). Clear divergence in expression patterns and motif structure was observed between the two genes. PtaLEA4-3 had the group-specific motif 1 repeated up to seven times, and its expression was observed in all of the tissues except phloem. The PtaLEA4-2 had only one repetitions of motif 1 that did not express in any tissue we studied. The enhanced salt-tolerance of PtaLEA4-3 with more motif repetitions was consistent with a previous study which investigated the repeat region (a highly conserved 22-mer motif) in the PM2 (LEA3) protein in E. coli. They have found that the recombinant strains with more repeat motifs had much higher viability ratios than the recombinant strains with shortened repeat motifs ${ }^{61}$. This evidence proved the fact that the conserved repeat motifs in LEA protein have played important function in salt tolerance.

The dehydrin group, also referred to as dehydrins, is the second largest group in the LEA family. Within this group, the dehydrin-5 induced a higher viability ratio to salt treatment, and dehydrin-4 increased the heat tolerance, while dehydrin-2 responded to both treatments. The $P$. tabuliformis proteins share the highly conserved motif elements of K-segment and S-segment, but they lack the Y-segement [(V/T) DEYGNP], which consistent with the report that this segment only been described in angiosperm ${ }^{62}$. The K-segments are found located near the $\mathrm{C}$-terminal region and supposed to be involved in the formation of class A2 amphipathic $\alpha$-helix ${ }^{63}$. The helix is considered to establish hydrophobic interactions with other proteins in order to stabilize cell membranes ${ }^{64}$. Thus, the role of the K-segment may be hydropobic interaction with partially denatured proteins and protect the cell membranes, especially under the stressed condition ${ }^{65,66}$. Another important S-segment (a tract of Ser-residues) is the motif $S$ in our analysis and it has been demonstrated that Ser-residues in the segment can be phosphorylated and leads to calcium binding ${ }^{67}$. The phosphorylation is also related to the binding of nuclear localization signal peptides to nuclear transport ${ }^{65}$. These motifs repetitions are variable in dehydrin proteins, especially in gymnosperms compared to the angiosperms. The dehydrin-5 lack the motif S, dehydrin-4 has three repetition of motif $\mathrm{K}$, while dehydrin-2 has only one motif $\mathrm{K}$. A recent study also reported novel conserved segments are associated with differential expression patterns in Pinus pinaster ${ }^{68}$. In that study the discovered novel conserved A - segment (EAASYYP) and E- segment (GHGYEGQFTPEEAEQQKH) which also could be found in dehydrin $-2 / 3 / 4 / 6$ in our study but could not be found in dehydrin-5. These diversified motif structures presumably related to the divergent tolerance abilities observed but the detailed underlying mechanisms remain to be explored.

\section{References}

1. Serrano, R. \& Rodriguez-Navarro, A. Ion homeostasis during salt stress in plants. Curr. Opin. Cell Biol. 13, 399-404 (2001).

2. Zhu, J. K. Plant salt tolerance. Trends Plant Sci. 6, 66-71 (2001).

3. Heremans, K. \& Smeller, L. Protein structure and dynamics at high pressure. BBA-Protein struct. M. 1386, 353-370 (1998).

4. Hazel, J. R. Thermal adaptation in biological membranes: is homeoviscous adaptation the explanation? Annu. Rev. Physiol. 57, 19-42 (1995).

5. Berry, J. \& Bjorkman, O. Photosynthetic response and adaptation to temperature in higher plants. Annu. Rev.Physiol. 31, 491-543 (1980).

6. Howarth, C. J. Genetic improvements of tolerance to high temperature. (Howarth Press, 2005).

7. Steponkus, P. L. Responses to extreme temperatures. Cellular and sub-cellular bases. Encyclopedia of Plant Physiology 12, 371-402 (1981).

8. Wang, W. X., Vinocur, B. \& Altman, A. Plant responses to drought, salinity and extreme temperatures: towards genetic engineering for stress tolerance. Planta 218, 1-14 (2003).

9. Tolleter, D., Hincha, D. K. \& Macherel, D. A mitochondrial late embryogenesis abundant protein stabilizes model membranes in the dry state. BBA-Gen. subjects 1798, 1926-1933 (2010).

10. Hincha, D. K. \& Thalhammer, A. LEA proteins: IDPs with versatile functions in cellular dehydration tolerance. Biochem. Soc. T. 40, 1000-1003 (2012).

11. Cuming, A. C. LEA proteins. (Kluwer press, 1999).

12. Galau, G. A., Hughes, D. W. \& Dure, L. Abscisic acid induction of cloned cotton late embryogenesis-bundant (Lea) mRNAs. Plant Mol. Biol. 7, 155-169 (1986).

13. Goyal, K., Browne, J. A., Burnell, A. M. \& Tunnacliffe, A. Dehydration-induced tps gene transcripts from an anhydrobiotic nematode contain novel spliced leaders and encode atypical GT-20 family proteins. Biochimie 87, 565-574 (2005).

14. Battista, J. R., Park, M. J. \& Mclemore, A. E. Inactivation of two homologues of proteins presumed to be involved in the desiccation tolerance of plants sensitizes Deinococcus radiodurans R1 to desiccation. Cryobiology 43, 133-139 (2001).

15. Hand, S. C., Jones, D., Menze, M. W. \& Witt, T. L. Life without water: expression of plant LEA genes by an anhydrobiotic arthropod. J. Exp. Zool. 305, 1-5 (2006).

16. Roberts, J. K., DeSimone, N. A., Lingle, W. L. \& Dure, L. Cellular concentrations and uniformity of cell-type accumulation of two LEA proteins in cotton embryos. Plant Cell 5, 769-781 (1993).

17. Silveira, V. et al. Endogenous abscisic acid and protein contents during seed development of Araucaia angustifolia. Biol. Plantarum 52, 101-104 (2008).

18. Wise, M. J. LEAping to conclusions: a computational reanalysis of late embryogenesis abundant proteins and their possible roles. BMC Bioinformatics 4, 52 (2003).

19. Serrano, R. \& Montesinos, C. Molecular bases of desiccation tolerance in plant cells and potential applications in food dehydration. Food Sci. Technol. Int. 9, 157-161 (2003).

20. Grelet, J. B. A. et al. Identification in pea seed mitochondria of a late-embryogenesis abundant protein able to protect enzymes from drying. Plant Physiol. 137, 157-167 (2005).

21. Shao, H. B., Liang, Z. S. \& Shao, M. A. LEA proteins in higher plants: structure, function, gene expression and regulation. Colloid. Surface B. 45, 131-135 (2005).

22. Bray, E. A. Molecular responses to water deficit. Plant Physiol. 103, 1035-1040 (1993).

23. Umezawa, T. et al. Engineering drought tolerance in plants: discovering and tailoring genes unlock the future. Curr. Opin. Biotech. 17, 113-122 (2006).

24. Ting, L., Jie, G. \& Qing-Yin, Z. Genome-wide analysis of the LEA (late embryogenesis abundant) protein gene family in Populus trichocarpa. Tree Genet. Genomes 9, 253-264 (2013).

25. Hundertmark, M. \& Hincha, D. K. LEA (late embryogenesis abundant) proteins and their encoding genes in Arabidopsis thaliana. BMC Genomics 9,118-139 (2008). 
26. Wang, X. S. et al. Genome-scale identification and analysis of LEA genes in rice (Oryza sativa L.). Plant Sci. 172, 414-420 (2007).

27. Choi, D. W., Zhu, B. \& Close, T. J. The barley (Hordeum vulgare L.) dehydrin multigene family: sequences, allele types, chromosome assignments, and expression characteristics of 11 Dhn genes of cv Dicktoo. Theor. Appl. Genet. 98, 1234-1247 (2007).

28. Du, D. L. et al. Genome-wide identification and analysis of late embryogenesis abundant (LEA) genes in Prunus mume. Mol. Biol. Rep. 40, 1937-1946 (2013).

29. Cao, J. \& Li, X. Identification and phylogenetic analysis of late embryogenesis abundant proteins family in tomato (Solanum lycopersicum). Planta 241, 757-772 (2015).

30. Charfeddine, S. et al. Genome-wide identification and expression profiling of the late embryogenesis abundant genes in potato with emphasis on dehydrins. Mol. Biol. Rep. 42, 1163-1174 (2015).

31. Liang, D., Xia, H., Wu, S. \& Ma, F. W. Genome-wide identification and expression profiling of dehydrin gene family in Malus domestica. Mol. Biol. Rep. 39, 10759-10768 (2012).

32. Battaglia, M. \& Covarrubias, A. A. Late embryogenesis abundant (LEA) proteins in legumes. Front. Plant Sci. 4190 doi: 10.3389/ fpls.2013.00190 (2013).

33. Jin-Zhuo, D. \& David, I. D. Cloning and characterization of six embryogenesis-associated cDNAs from somatic embryos of Picea glauca and their comparative expression during zygotic embryogenesis. Plant Mol. Biol. 39 859-864 (1998).

34. Jarvis, S. B., Taylor, M. A., Macleod, M. R. \& David, H. V. Cloning and characterization of the cDNA clones of three genes that are differentially expressed during dormancy-breakage in the seeds of Douglas fir (Pseudotsuga menziesii). J. Plant Physiol. 147, 559-566 (1996).

35. Perdiguero, P., Collada, C. \& Soto, A. Novel dehydrins lacking complete K-segments in Pinaceae, the exception rather than the rule. Front. Plant Sci. 5, 682. doi: $10.3389 /$ fpls.2014.00682 (2014).

36. Mao, J. F. \& Wang, X. R. Distinct niche divergence characterizes the homoploid hybrid speciation of Pinus densata on the Tibetan plateau. Am. Nat. 177, 424-439 (2011).

37. Wang, B. F. et al. Verification of the resistance of a LEA gene from Tamarix expression in Saccharomyces to aboiotic stresses. Journal of Forestry Research 19, 58-62 (2008).

38. Lei, Z., Akinori, O., Masamichi, T. \& Ryozo, I. Expression of plant group 2 and group 3 lea genes in Saccharomyces cerevisiae revealed functional divergence among LEA proteins. J. Biochem. 127, 611-616 (2000).

39. Honjoh, K. et al. Introduction of the hiC6 gene, which encodes a homologue of a late embryogenesis abundant (LEA) protein, enhances freezing tolerance of yeast. J. Plant Physiol. 155, 509-512 (1999).

40. Paul, A., Singh, S., Sharma, S. \& Kumar, S. A stress-responsive late embryogenesis abundant protein 7 (CsLEA7) of tea [Camellia sinensis (L.) O. Kuntze] encodes for a chaperone that imparts tolerance to Escherichia coli against stresses. Mol. Biol. Rep. 41, 7191-7200 (2014).

41. Dang, N. X., Popova, A. V., Hundertmark, M. \& Hincha, D. K. Functional characterization of selected LEA proteins from Arabidopsis thaliana in yeast and in vitro. Planta 240, 325-336 (2014).

42. Thompson, J. D. et al. The CLUSTAL_X windows interface: flexible strategies for multiple sequence alignment aided by quality analysis tools. Nucleic Acids Res. 25, 4876-4882 (1997).

43. Hall, T. A. BioEdit: a user-friendly biological sequence alignment editor and analysis program for Windows 95/98/NT. Nucleic Acids Symposium Series 41, 4 (1999).

44. Tamura, K. et al. MEGA5: Molecular evolutionary genetics analysis using maximum likelihood, evolutionary distance and maximum parsimony methods. Mol. Biol. Evol. 28, 2731-2738 (2011).

45. Bailey, T. L. \& Elkan, C. Fitting a mixture model by expectation maximization to discover motifs in biopolymers. Proc. Int. Conf. Intell. Syst. Mol. Biol. 2, 28-36 (1994).

46. Emanuelsson, O., Nielsen, H., Brunak, S. \& Von, H. G. Predicting subcellular localization of proteins based on their N-terminal amino acid sequence. J. Mol. Biol. 300, 1005-1016 (2000).

47. Martha, S. V. \& Anne, E. C. Using cellprofiler for automatic identification and measurement of biological objects in images. Current Protocols in Molecular Biology 109, 1-13 (2008).

48. Bies-Etheve, N. et al. Inventory, evolution and expression profiling diversity of the LEA (late embryogenesis abundant) protein gene family in Arabidopsis thaliana. Plant Mol. Biol. 67, 107-124 (2008).

49. Du, D. L. et al. Genome-wide identification and analysis of late embryogenesis abundant (LEA) genes in Prunus mume. Mol. Biol. Rep. 40, 1937-1946 (2013).

50. Liang, D., Xia, H., Wu, S. \& Ma, F. W. Genome-wide identification and expression profiling of dehydrin gene family in Malus domestica. Mol. Biol. Rep. 39, 10759-10768 (2012).

51. Xu, D. P., Duan, X. L. \& Wang, B. Y. Expression of a late embryogenesis abundant protein gene HVA1, from barley confers tolerance to water deficit and salt stress in transgenic rice. Plant Physiol. 110, 249-257 (1996).

52. Zhang, L., Ohta, A., Takagi, M. \& Imai, R. Expression of plant group 2 and group 3 lea genes in Saccharomyces cerevisiae revealed functional divergence among LEA proteins. J. Biochem. 127, 611-616 (2000).

53. Dalal, M., Tayal, D., Chinnusamy, V. \& Bansal, K. C. Abiotic stress and ABA-inducible group 4 LEA from Brassica napus plays a key role in salt and trought tolerance. J. Biotechnol. 139, 137-145 (2009).

54. Tang, J. \& Lin, N. F. Some problems of ecological environmental geology in arid and semiarid areas of China. Environ. Geol. 26, 64-67(1995).

55. Tang, J. \& Lin, N. F. Study on the environment evolution and the analysis of causes to land salinization and desertification in songnen plain. Quaternary Sciences 04, 474-483(2005).

56. Li, X. Y. et al. Assessment for salinized wasteland expansion and land use change using GIS and remote sensing in the west part of northeast China. Environ. Monit. Assess 131, 421-437(2007)

57. Sidari, M., Mallamaci, C. \& Muscolo, A. Drought, salinity and heat differently affect seed germination of Pinus pinea. J. Forest Res. 13, 326-330(2008).

58. Hundertmark, M., Buitink, J., Leprince, O. \& Hincha, D. K. The reduction of seed-specific dehydrins reduces seed longevity in Arabidopsis thaliana. Seed Sci. Res. 21, 165-173 (2011).

59. Ting, L. et al. Extensive functional diversification of the Populus Glutathione S-Transferase supergene family. Plant Cell 21, 3749-3766 (2009).

60. Ying, L., Dan, C. \& Yi-Zhi, Z. Expression in Escherichia coli of three different soybean late embryogenesis abundant genes to investigate enchanced stress tolerance. J. Integr. Plant Biol. 47,613-621 (2005).

61. Liu, Y. \& Zheng, Y. Z. PM2, a group 3 LEA protein from soybean, and its 22-mer repeating region confer salt tolerance in Escherichia coli. Biochem. Bioph. Res. Co. 331, 325-332 (2005).

62. Perdiguero, P. et al. Novel dehydrins lacking complete K-segments in Pinaceae. The exception rather than the rule. Front. Plant Sci. 5, $682(2014)$.

63. Baker, J., DennsSteele, C. \& Dure, L. Sequence and characterization of 6 Lea proteins and their genes from cotton. Plant Mol. Biol. 11, 277-291(1988).

64. Koag, M. C., Fenton, R. D., Wilkens, S. \& Close, T. J. The binding of Maize DHN1 to lipid vesicles gain of structure and lipid specificity. Plant Physiol. 131, 309-316 (2003).

65. Close, T. J. Dehydrins: Emergence of a biochemical role of a family of plant dehydration proteins. Physiology Plant 97,795-803 (1996). 
66. Koag, M. C. et al. The K-segment of maize DHN1 mediates binding to anionic phospholipid vesicles and concomitant structural changes. Plant Physiol. 150, 1503-1514 (2009).

67. Alsheikh, M. K., Svensson, J. T. \& Randall, S. K. Phosphorylation regulated ion-binding is a property shared by the acidic subclass dehydrins. Plant cell Environ. 28, 1114-1122 (2005).

68. Perdiguero, P. \& Barbero, M. C. Novel conserved segments are associated with differential expression patterns for Pinaceae dehydrins. Planta 236, 1863-1874 (2012).

\section{Acknowledgements}

We would like to thank Prof. Chuck Canon form Texas Tech University USA and Dr. Joeri S.Strijk for valuable suggestions and English revision of the manuscript, Dr. Zhao Wei helping use the Cellprofiler software, Zhao $\mathrm{Li}$ and Yang Xue help with the experiment. This study was supported by grants from the National Science Foundation of China (NSFC, 31300560).

\section{Author Contributions}

J.G. and T.L. conceived and designed research. J.G. conducted experiments, analyzed data and wrote the manuscript. All authors read and approved the manuscript.

\section{Additional Information}

Supplementary information accompanies this paper at http://www.nature.com/srep

Competing financial interests: The authors declare no competing financial interests.

How to cite this article: Gao, J. and Lan, T. Functional characterization of the late embryogenesis abundant (LEA) protein gene family from Pinus tabuliformis (Pinaceae) in Escherichia coli. Sci. Rep. 6, 19467; doi: 10.1038/srep19467 (2016).

(c) (i) This work is licensed under a Creative Commons Attribution 4.0 International License. The images or other third party material in this article are included in the article's Creative Commons license, unless indicated otherwise in the credit line; if the material is not included under the Creative Commons license, users will need to obtain permission from the license holder to reproduce the material. To view a copy of this license, visit http://creativecommons.org/licenses/by/4.0/ 